\title{
EL PROYECTO DE VIDA Y LA RETENCIÓN DE JÓVENES TALENTO EN EL EJE CAFETERO, SANTANDERES Y VALLE DE ABURRÁ
}

\author{
Life project and retention young talents in the Eje Cafetero, Santanderes \\ and Valle de Aburra
}

\author{
Ángel Emilio Muñoz Cardona ${ }^{1}$ \\ ${ }^{1}$ Escuela Superior de Administración Pública \\ Contacto: $h$ ttps://orcid.org/0000-0001-5008-0983
}

(Recibido Noviembre 15 de 2018 y aceptado Agosto 12 de 2019)

\section{Resumen}

La finalidad del presente ensayo de investigación es demostrar la hipótesis "existe una relación positiva entre emprendimiento y felicidad como realización de los proyectos de vida que facilita el desarrollo local y la reducción de las migraciones de jóvenes talento en las áreas metropolitanas del Eje Cafetero, Santanderes y Valle de Aburrá". En otras palabras, ¿cómo ven los jóvenes universitarios el futuro laboral en sus municipalidades?, ¿qué confianza les brinda el crecimiento futuro de su municipio? La investigación se basa en los índices internacionales de: Bienestar Pluridimensional y de Felicidad Integral. Para tal efecto, se realizaron 1780 cuestionarios, bajo el método de análisis multivariado, con un nivel de confianza del 95\% y un margen de error del 5\%. El estudio concluye que Cúcuta, Medellín y Manizales son las ciudades que más retienen a sus jóvenes talento.

Palabras clave: felicidad subjetiva, felicidad intersubjetiva, equipamiento de ciudad, proyecto de vida

\begin{abstract}
The purpose of this research essay is to demonstrate the hypothesis "There is a positive relationship between entrepreneurship and happiness as a realization of life projects that facilitates local development and the reduction of migrations of talented young people in the metropolitan areas of Coffee Region, Santanderes and Aburra Valley." In other words, how doyoung university students see the future of work in their municipalities? What confidence does the future growth of their municipality offer them? The research is based on the international indexes of: Multi-dimensional Well-Being and Integral Happiness. For this purpose, 1780 questionnaires were carried out, using the multivariate analysis method, with a confidence level of $95 \%$ and a margin of error of $5 \%$. The study concludes that Cucuta, Medellin and Manizales are the cities that most retain theiryoung talent.
\end{abstract}

Key words: subjective happiness, intersubjective happiness, city equipment, Life project

\section{INTRODUCCIÓN}

Ante la discusión si existe o no la felicidad y si es posible medirla, los autores clásicos de la economía como Adam Smith (1997), [1-2], y más recientemente la escuela del utilitarismo [3-4] ven en la felicidad general de los ciudadanos de los países una suerte de buenas políticas de distribución económi$\mathrm{ca}$, de gobiernos menos corruptos y transparentes.

\begin{abstract}
De allí que el estudio del concepto de felicidad se ha dividido en dos corrientes: la felicidad subjetiva y la felicidad intersubjetiva.
\end{abstract}

La felicidad subjetiva da a entender la valoración individual que cada consumidor da al disfrute de bienes que demanda, valoración microeconómica que puede cambiar con los estados de ánimo o de riqueza de la persona en concreto, según el índice de felicidad [5]. 
Para el historiador Noah Harari, "lo subjetivo es algo que existe en función de la conciencia y creencia de un único individuo y desaparece o cambia si este individuo concreto cambia sus creencias" p. 136 [6]. Por el contrario, la felicidad intersubjetiva da cuenta de las relaciones sociales propias de los estados sociales de derecho; de la capacidad de conciliación, de establecer acuerdos, de asociación para la consecución del bien común y de investigación con fines sociales, como afirma Noah Harari, "Lo intersubjetivo es algo que existe en el seno de la red de comunicación que conecta la conciencia subjetiva de muchos individuos" p. 136 [6]. Desde esta perspectiva aparecen aportes significativos de economistas contemporáneos como Amartya Sen, Paul Fitoussi y Joseph Stiglitz, incluso de filósofos morales como Martha Nussbaum y François Dubet.

Si el bienestar de la población, es decir, la felicidad general nace de las inversiones públicas entonces la hipótesis de investigación parte de creer que "existe una relación positiva entre emprendimiento y felicidad como realización de los proyectos de vida que facilita el desarrollo local y la reducción de las migraciones de jóvenes talento en las áreas metropolitanas del Eje Cafetero, Santanderes y Valle de Aburrá". Para demostrar dicha hipótesis se realizaron 1780 encuestas a jóvenes de último semestre universitario de las principales universidades públicas y privadas de las regiones, analizadas bajo el método estadístico multivariado con un alfa del $95 \%$ de confianza y un margen de error del 5\%. En dichos cuestionarios se evalúo el equipamiento de ciudad, la calidad de vida, las necesidades básicas insatisfechas y los proyectos de vida de los jóvenes universitarios de último semestre [7]. En el presente ensayo de investigación solo tomará la variable proyectos de vida en relación con la satisfacción que tienen los jóvenes universitarios con la clase política, la administración pública y las expectativas de crecimiento económico en las diferentes ciudades de las áreas metropolitanas.

Si bien el orden social como político han sido transformados por los avances de la ciencia, se pregunta Yuval Noah en su libro De animales a dioses: ¿somos realmente más felices? ¿Es realmente el mundo un mejor lugar para vivir?
¿Fue más feliz Neil Armstrong, cuyas huellas permanecen intactas sobre la Luna carente de viento, que el cazador-recolector anónimo que hace 30.000 años dejó la huella de su mano en una pared de la cueva de Chauvet? Y, si no es así, ¿qué sentido ha tenido desarrollar la agricultura, las ciudades, la escritura, las monedas, la ciencia y la industria?, ¿cuál es el beneficio del capitalismo? p. 412 [6]

Para los investigadores de la felicidad [3-6] existe una correlación positiva entre capacidades humanas y felicidad, las cuales se han visto reflejadas en la disminución de enfermedades y aumentos de las edades promedios de vida, por ejemplo, la medicina moderna ha logrado reducir en los últimos doscientos años la mortalidad infantil del $33 \%$ a menos del $5 \%$. Desde el final de la segunda guerra mundial con la amenaza nuclear, el mundo ha vivido una cierta estabilidad de paz. Las confrontaciones son menos frecuentes; los organismos internacionales y las asociaciones estatales intervienen a favor de salidas conciliadas, reducciones del conflicto. Evitando que el mundo se destruya de manera escalonada [6].

Otros investigadores de la escuela latinoamericana de la Filosofía de la Liberación ven en la correlación de las variables capacidades humanas y felicidad una relación negativa; una apuesta egoísta de poder en la que naciones y comunidades más fuertes buscan someter a otras, expropiándola de sus riquezas naturales [8]. Conciben el desarrollo y el subdesarrollo como una colonización, es decir, como un saqueo de las riquezas mineras y de hidrocarburos del segundo a favor del primero. Riquezas de explotación productiva que hacen más felices a ciudadanos de pocos países que a miles de millones de ciudadanos de otras naciones que lo pierden todo, incluso hasta las esperanzas de un futuro o posibilidades de una mejor vida. Sin embargo, dichas relaciones de la política económica internacional que profundizan las brechas sociales de desarrollo y subdesarrollo económico hacen posible medir la felicidad, tal y como [9] y Robert Kaplan en su libro "La venganza de la geografía" lo ilustra.

Hechas las anteriores claridades sobre el concepto 
de felicidad pasemos a indagar sobre la satisfacción de los jóvenes universitarios de último semestre del Eje Cafetero con el gobierno, la policía, la clase política y el progreso económico de la región.

\section{Eje Cafetero}

En el Eje Cafetero se realizaron 385 encuestas a jóvenes universitarios de últimos semestres de las ciudades de Armenia, Manizales y Pereira, tanto de universidades públicas como privadas.

Satisfacción con el gobierno y el progreso de económico de la ciudad

Una buena administración pública, en términos aristotélicos, consiste en hacer de la política el bien supremo, y "ésta pone el máximo empeño en hacer a los ciudadanos de una cierta cualidad y buenos e inclinados a hacer el bien" [10] es decir, procurar el bien para sí y para los demás, de alcanzar la virtud social. Y para ello todo ser humano busca hacer realidad sus sueños, de crecer en su humanidad social junto con los otros, de allí "que sea razonable no llamar feliz a un buey, un caballo o ningún otro animal, pues ninguno de ellos es capaz de participar en una actividad semejante" [10]

En este sentido, la mala administración del gobierno es la fuente de todos los males sociales, que allana de pobreza moral, económica y social a todos sus ciudadanos hasta acabar con su calidad de seres libres [4-11]. Comencemos, por preguntarnos, ¿cómo califican los jóvenes universitarios sus gobiernos?, ¿cómo ven el futuro de sus regiones para la realización de sus proyectos de vida?

El 19,1\% de los jóvenes universitarios de último semestre del área metropolitana de Armenia, el 30,6\% de Manizales y el $50 \%$ de Pereira califican la gestión del alcalde de satisfactoria y muy satisfactoria. Es decir, de muy aceptable, así lo afirman los universitarios encuestados de Pereira; por el contrario, el 47,7\% del área metropolitana de Armenia están prioritariamente insatisfechos y muy insatisfechos. Del total general, el 37,2\% de los jóvenes universitarios encuestados del Eje Cafetero están regularmente satisfechos con sus gobiernos de ciudad (Ver Tabla 1).

En cuanto a la clase política el 33,9\% de los jóvenes universitarios del área metropolitana de Pereira, el 24,5\% de Manizales y el 15,9\% de Armenia están satisfechos y muy satisfechos. La buena aceptación que tienen los universitarios de último semestre de Pereira con su alcalde influye positivamente en la aceptación de los líderes políticos de la ciudad. En el caso de Armenia ocurre exactamente igual, la baja satisfacción de los universitarios con la gestión del alcalde es igualmente causa de insatisfacción con la clase política del municipio en un 57,1\% (Ver Tabla 1).

Tabla 1. Eje Cafetero. Satisfacción con el gobierno de la ciudad.

\begin{tabular}{ccccc}
\hline \multicolumn{5}{c}{ Nivel de satisfacción con la gestión del } \\
alcalde \\
\hline $\begin{array}{c}\text { Áreas } \\
\begin{array}{c}\text { metropo- } \\
\text { litanas }\end{array}\end{array}$ & Armenia & Manizales & Pereira & Total \\
\hline 1 & $30.2 \%$ & $13.6 \%$ & $5.4 \%$ & $15.1 \%$ \\
2 & $17.5 \%$ & $16.6 \%$ & $12.5 \%$ & $16.1 \%$ \\
3 & $33.3 \%$ & $39.2 \%$ & $32.1 \%$ & $37.2 \%$ \\
4 & $15.9 \%$ & $24.2 \%$ & $37.5 \%$ & $24.7 \%$ \\
5 & $3.2 \%$ & $6.4 \%$ & $12.5 \%$ & $6.8 \%$ \\
\hline \multicolumn{5}{c}{ Nivel de satisfacción con la clase política } \\
\hline 1 & $38.1 \%$ & $18.9 \%$ & $8.9 \%$ & $20.6 \%$ \\
2 & $19.0 \%$ & $20.8 \%$ & $19.6 \%$ & $20.3 \%$ \\
3 & $27.0 \%$ & $35.8 \%$ & $37.5 \%$ & $34.6 \%$ \\
4 & $12.7 \%$ & $20.0 \%$ & $26.8 \%$ & $19.8 \%$ \\
5 & $3.2 \%$ & $4.5 \%$ & $7.1 \%$ & $4.7 \%$ \\
\hline
\end{tabular}

Fuente: Centro de estudios económicos regionales CEER de la ESAP y del grupo de investigación Gobierno, Territorio y Cultura. 1 es poco satisfecho. 5 es muy satisfecho. 3 regularmente satisfecho. 2018.

Manizales y Pereira, son las ciudades que cuentan con la mayor satisfacción de gobierno, gracias a la buena gestión 
de sus alcaldes. Apreciación de los jóvenes universitarios encuestados que lleva a la pregunta: ¿Qué tan satisfecho está con el progreso económico de su municipio?

\begin{tabular}{|c|c|c|c|c|}
\hline $\begin{array}{l}\text { Nivel de } \\
\text { satisfac- } \\
\text { ción }\end{array}$ & Armenia & Manizales & Pereira & $\begin{array}{l}\text { Total } \\
\text { general }\end{array}$ \\
\hline 1 & $21.0 \%$ & $11.3 \%$ & $8.9 \%$ & $12.5 \%$ \\
\hline 2 & $29.0 \%$ & $9.4 \%$ & $16.1 \%$ & $13.6 \%$ \\
\hline 3 & $30.6 \%$ & $41.5 \%$ & $28.6 \%$ & $37.9 \%$ \\
\hline 4 & $16.1 \%$ & $30.6 \%$ & $39.3 \%$ & $29.5 \%$ \\
\hline 5 & $3.2 \%$ & $7.2 \%$ & $7.1 \%$ & $6.5 \%$ \\
\hline $\begin{array}{c}\text { Total } \\
\text { general }\end{array}$ & $100.0 \%$ & $100.0 \%$ & $100.0 \%$ & $100.0 \%$ \\
\hline
\end{tabular}

Fuente: Centro de estudios económicos regionales CEER de la ESAP y del grupo de investigación Gobierno, Territorio y Cultura. 1 es poco satisfecho. 5 es muy satisfecho. 3 regularmente satisfecho. 2018.

Según la Tabla 2, el 19,3\% de los jóvenes universitarios de último semestre de Armenia, el 37,8\% de Manizales y el $46,4 \%$ de Pereira están satisfechos y muy satisfechos con el progreso económico de sus ciudades. En otras palabras, existe una correlación positiva entre la percepción de satisfacción de los ciudadanos con el gobierno local y el progreso económico.

Entre más alta es la confianza de los futuros profesionales en la gestión del alcalde mayor es la satisfacción con el progreso económico de la ciudad, así lo demuestra el estudio de investigación en "Felicidad Urbana" y "Manual de civismo" [12-13]. Pereira, por ejemplo, es la ciudad con mejor percepción de progreso económico y con mayor satisfacción en la gestión del alcalde. De igual manera, ocurre para Manizales (Ver Tabla 2). Asimismo, se espera que a mayor confianza de los ciudadanos en el progreso económico de sus ciudades, es menor la migración de los jóvenes universitarios de último semestre, es decir, menor es la Migración Altamente Calificada MAC, en otras palabras, más alta es la tendencia a permanecer en la ciudad realizando su proyecto de vida.

Esta tendencia se mantiene, cuando las ciudades del Eje Cafetero son ciudades relativamente seguras, en la que los jóvenes de formación calificada afirman confiar en las acciones de seguridad de la policía el 35,4\%, las califican de buenas, el $43,2 \%$ de regular y el $16,7 \%$ de mala. Es decir, la gente confía en las acciones realizadas por las instituciones de la policía en su ciudad, lo que facilita los apegos ciudadanos a su territorio y a su localidad (Ver Tabla 2).

Ante la pregunta a los futuros profesionales del Eje Cafetero: ¿posee usted algún proyecto de vida? El 80,7\% dijo SI y el 19,3\% dijo NO. Y ante la pregunta ¿cuál es su proyecto de vida? El 53,4\% de los universitarios del área metropolitana de Armenia, el 40,8\% de Manizales y el $32,3 \%$ de Pereira respondieron ser profesionales, pero con la diferencia de que los jóvenes universitarios de Pereira en un 11,3\% y los de Manizales en un 14,2\% dijeron querer ser emprendedores, lo que fácilmente puede significar mayores facilidades de crecimiento económico, mayor diversidad productiva y considerables fuentes de empleo, es decir, las ciudades de Pereira y Manizales desde su infraestructura estimulan el crecimiento empresarial; la ciudad de Armenia no genera las mismas expectativas en sus futuros profesionales (Ver Tabla 3).

Según la Tabla 3, el 56,7\% de los jóvenes universitarios de último semestre desean realizar su proyecto de vida en sus ciudades, porque ven en el Eje Cafetero un adecuado desarrollo municipalista, afirmó el $21,3 \%$ de los encuestados. Sin embargo, el $19,2 \%$ no contesto la pregunta, lo que abre un gran margen de duda sobre las razones del por qué. El 89,4\% de los jóvenes que respondieron no realizar el proyecto de vida en sus ciudades el $50 \%$ de ellos afirman regresar nuevamente a trabajar y a vivir. Respuesta que fortalece la hipótesis de investigación sobre el desarrollo socioeconómico y la retención de los jóvenes talentosos MAC.

De acuerdo a las respuestas de los futuros profesionales 
encuestados, se puede afirmar que El Eje Cafetero retiene, en promedio el $42,2 \%$ de los jóvenes que forma. Manizales logra retener, aproximadamente, el $51,8 \%$, Pereira el $35,7 \%$ y Armenia el $39,1 \%$.

Tabla 3. Eje Cafetero. Realización del proyecto de vida.

\begin{tabular}{|c|c|c|c|c|c|c|}
\hline $\begin{array}{l}\text { ¿Puede realizar su proyecto de } \\
\text { vida en su municipio? }\end{array}$ & \multicolumn{3}{|c|}{ Hombres } & \multicolumn{2}{|c|}{ Mujeres } & Total \\
\hline $\mathrm{SI}$ & \multicolumn{3}{|c|}{$53.7 \%$} & \multicolumn{2}{|c|}{$59.4 \%$} & $56.7 \%$ \\
\hline NO & \multicolumn{3}{|c|}{$46.3 \%$} & \multicolumn{2}{|c|}{$40.6 \%$} & $43.3 \%$ \\
\hline \multicolumn{5}{|l|}{ ¿Por qué Si puede realizarlo? } & & \\
\hline Adecuado desarrollo municipal & \multicolumn{3}{|c|}{$20.4 \%$} & \multicolumn{2}{|c|}{$22.2 \%$} & $21.3 \%$ \\
\hline Amor por el territorio & \multicolumn{3}{|c|}{$4.7 \%$} & \multicolumn{2}{|c|}{$3.1 \%$} & $3.9 \%$ \\
\hline Buena infraestructura educativa & \multicolumn{3}{|c|}{$4.7 \%$} & \multicolumn{2}{|c|}{$6.2 \%$} & $5.5 \%$ \\
\hline \multicolumn{7}{|l|}{ ¿Por qué No puede realizarlo? } \\
\hline Insatisfacción por el territorio & \multicolumn{3}{|c|}{$12.0 \%$} & \multicolumn{2}{|c|}{$9.3 \%$} & $10.6 \%$ \\
\hline Bajo desarrollo municipal & \multicolumn{3}{|c|}{$12.0 \%$} & \multicolumn{2}{|c|}{$19.6 \%$} & $15.8 \%$ \\
\hline No contesta & \multicolumn{3}{|c|}{$46.1 \%$} & \multicolumn{2}{|c|}{$39.7 \%$} & $42.9 \%$ \\
\hline \multicolumn{7}{|c|}{ Volveria a su municipio a } \\
\hline & Trabajar & Pasear & Vivir & No volveria & No contesta & Total \\
\hline Armenia & $12.5 \%$ & $39.1 \%$ & $26.6 \%$ & $0.0 \%$ & $21.9 \%$ & $100.0 \%$ \\
\hline Manizales & $23.5 \%$ & $29.4 \%$ & $28.3 \%$ & $1.4 \%$ & $17.4 \%$ & $100.0 \%$ \\
\hline Pereira & $14.3 \%$ & $35.7 \%$ & $21.4 \%$ & $3.6 \%$ & $25.0 \%$ & $100.0 \%$ \\
\hline \multicolumn{7}{|c|}{ ¿Cuál es su proyecto de vida? } \\
\hline & $\begin{array}{l}\text { Proyecto } \\
\text { Profesional }\end{array}$ & $\begin{array}{l}\text { Proyecto } \\
\text { Familiar }\end{array}$ & $\begin{array}{l}\text { Proyecto } \\
\text { Laboral }\end{array}$ & $\begin{array}{l}\text { Emprendi- } \\
\text { miento }\end{array}$ & No contesta & Total \\
\hline Hombres & $16.9 \%$ & $2.7 \%$ & $5.0 \%$ & $6.8 \%$ & $16.9 \%$ & $48.2 \%$ \\
\hline Mujeres & $24.8 \%$ & $3.4 \%$ & $6.1 \%$ & $5.9 \%$ & $11.7 \%$ & $51.8 \%$ \\
\hline Total & $41.7 \%$ & $6.1 \%$ & $11.0 \%$ & $12.6 \%$ & $28.6 \%$ & $100.0 \%$ \\
\hline
\end{tabular}

Fuente: Centro de estudios económicos regionales CEER de la ESAP y del grupo de investigación Gobierno, Territorio y Cultura. 2018.

Ahora bien, respecto al estudio de los jóvenes de último semestre de las áreas metropolitanas de Cúcuta y Bucaramanga, se realizaron 340 encuestas entre las distintas universidades de la región, tanto públicas como privadas.

\section{Santanderes}

A través del presente estudio se observará si el estudio del 2018 de La Encuesta de Percepción Ciudadana Comparada EPCC sobre calidad de vida y movilidad se equi para con el de Felicidad Urbana que hiciera la Escuela Superior de Administración Pública en el mismo año [14].

Según la EPCC, Cúcuta es una de las ciudades más afectadas por las políticas del gobierno de Venezuela que ha dado paso al éxodo de miles de venezolanos al país. Decisiones de política gubernamental venezolana que han afectado la economía de Cúcuta y su área metropolitana con más fuerza en los dos últimos años. 
Según indicadores económicos regionales para el primer semestre de 2018, entregados por la Cámara de Comercio de Cúcuta, el precio de los alimentos y de la salud fueron los que más aumentaron [14]. Sin embargo, el IPC de Cúcuta y su área metropolitana fue el más bajo en contraste con otras ciudades como Bogotá, Manizales y Cali; es decir, Cúcuta es la séptima ciudad del país con menor costo de vida, lo que puede obedecer al estímulo generado por los crecimientos del mercado [15]. No obstante, cabe anotar que la economía del área metropolitana de Cúcuta se basa, principalmente, en la exportación de combustibles fósiles en más de un $71 \%$, y un $12 \%$ en cobre, almidón y café. El $81 \%$ de las exportaciones se realizan por vía marítima a países como Venezuela, Turquía y Ecuador. El total de las exportaciones del área metropolitana de Cúcuta representan menos del 1\% del total de las exportaciones del país, lo que muestra una baja diversidad productiva y de transformación de productos finales.

La economía interna del área metropolitana se basa, primordialmente, en el comercio y está muy afectada por el comercio ilegal de gasolina que representa más del $41 \%$ de la producción total. Es decir, casi el 50\% de la gasolina que se consume en Cúcuta es de contrabando, lo que perjudica los recursos fiscales municipales y nacionales para la inversión y las políticas de distribución. Pero quizás, lo más grave son las fuentes de empleo que genera la práctica generalizada del contrabando de combustible [14].

Satisfacción con el gobierno y el progreso de económico de la ciudad

En las áreas metropolitanas de Cúcuta y Bucaramanga los jóvenes universitarios de último semestre califican la seguridad en las acciones de la policía de muy regulares. El 59,8\% las califican de regulares y malas, incluso el $2.9 \%$ de los estudiantes dicen que son inexistentes.

Para los estudiantes encuestados del área metropolitana de Cúcuta son regulares, ya que para el $49,4 \%$ son regulares y malas, y para el 3,4\% inexistente. De allí, que un poco más del $50 \%$ de los estudiantes de último semestre universitario encuestados afirmen estar regularmente satisfechos e insatisfechos con la seguridad de las zonas en que ellos viven.

Tabla 4. Santanderes. Calidad con la seguridad.

\begin{tabular}{ccc}
\hline $\begin{array}{c}\text { Satisfacción con la } \\
\text { seguridad }\end{array}$ & $\begin{array}{c}\text { Área de } \\
\text { Bucaramanga }\end{array}$ & Área de Cúcuta \\
\hline 1 (Muy insatisfecho) & $1.4 \%$ & $2.3 \%$. \\
2 & $18.6 \%$ & $25.6 \%$ \\
3 & $34.9 \%$ & $23.3 \%$ \\
4 & $38.6 \%$ & $33.7 \%$ \\
5 (Muy satisfecho) & $6.6 \%$ & $15.1 \%$ \\
\hline Total general & $\mathbf{1 0 0 . 0 \%}$ & $\mathbf{1 0 0 . 0 \%}$ \\
\hline
\end{tabular}

Fuente: Centro de estudios económicos regionales CEER de la ESAP y del grupo de investigación Gobierno, Territorio y Cultura de Antioquia. 2018. .

El $45,2 \%$ de los estudiantes encuestados del área metropolitana de Bucaramanga y el $48,8 \%$ del área metropolitana de Cúcuta afirman estar satisfechos y muy satisfechos con la seguridad de la zona (Ver Tabla 4). Datos de investigación que alertan a la administración pública para mejorar la seguridad local y la apreciación que los estudiantes tienen de sus gendarmes.

En cuanto a la satisfacción con la gestión del gobierno municipal y con la calidad del gobierno municipal, los estudiantes de último semestre del área metropolitana de Cúcuta las evalúan de buenas y regulares. Es decir, las aprueban en mayor porcentaje en un 58,4\%. Por el contrario, los estudiantes encuestados del área metropolitana de Bucaramanga solo las aprueban un 38,9\%.

Para el $61,5 \%$ de los bumangueses la calidad del gobierno municipal es principalmente regular y mala (Ver Tabla 5). La razón principal está en cómo ellos perciben el progreso económico de la región. El 40,2\% y 48,3\% de los estudiantes encuestados del área metropolitana de Bucaramanga y de Cúcuta, respectivamente, están satisfechos y muy satisfechos con el progreso económico 
de sus municipalidades; no obstante, la otra mitad afirma estar insatisfecha. Estas diferencias de satisfacción e insatisfacción determinan y evalúan la gestión del alcalde, y a su vez, en la misma correlación, la de la clase política de las diferentes municipalidades (Ver Tabla 5).

Tabla 5. Santanderes. Satisfacción con el gobierno

\begin{tabular}{ccccc}
\hline \multicolumn{2}{c}{ Gestión del alcalde } & \multicolumn{2}{c}{ Clase Política } \\
\hline $\begin{array}{c}\text { Evalua- } \\
\text { ción }\end{array}$ & $\begin{array}{c}\text { Área de } \\
\text { Bucara- } \\
\text { manga }\end{array}$ & $\begin{array}{c}\text { Área de } \\
\text { Cúcuta }\end{array}$ & $\begin{array}{c}\text { Área de } \\
\text { Bucara- } \\
\text { manga }\end{array}$ & $\begin{array}{c}\text { Área de } \\
\text { Cúcuta }\end{array}$ \\
\hline 1 & $4.8 \%$ & $5.6 \%$ & $15.1 \%$ & $16.9 \%$ \\
2 & $23.1 \%$ & $27.0 \%$ & $23.6 \%$ & $22.5 \%$ \\
3 & $33.6 \%$ & $25.8 \%$ & $37.6 \%$ & $24.7 \%$ \\
4 & $23.4 \%$ & $23.6 \%$ & $13.7 \%$ & $22.5 \%$ \\
5 & $15.1 \%$ & $18.0 \%$ & $10.0 \%$ & $13.5 \%$ \\
\hline $\begin{array}{c}\text { Total } \\
\text { general }\end{array}$ & $\mathbf{1 0 0 . 0 \%}$ & $\mathbf{1 0 0 . 0 \%}$ & $\mathbf{1 0 0 . 0 \%}$ & $\mathbf{1 0 0 . 0 \%}$ \\
\hline
\end{tabular}

Fuente: Centro de estudios económicos regionales CEER de la ESAP y del grupo de investigación Gobierno, Territorio y Cultura de Antioquia. 1 es poco satisfecho. 5 es muy satisfecho. 3 regularmente satisfecho. 2018.

Dado que los estudiantes de último semestre del área metropolitana de Bucaramanga perciben un bajo crecimiento económico de sus municipalidades, el 61,5\% evalúan la gestión de sus alcaldes de regular e insatisfactoria. Por el contrario, en un menor porcentaje, el 58,4\% de los estudiantes encuestados del área metropolitana de Cúcuta evalúan la gestión de sus alcaldes de regular e insatisfactoria. En otras palabras, los jóvenes universitarios de último semestre de Cúcuta, se encuentran regularmente satisfechos con la gestión de sus alcaldes y de su clase política. Los estudiantes de encuestados de Bucaramanga afirman estar más satisfechos (Ver Tabla 5).

Satisfacción con el proyecto de vida.

Conocidas las percepciones de los estudiantes de último semestre universitario sobre el progreso económico de sus municipalidades, la calidad de sus gobiernos, la satisfacción con la gestión de los alcaldes y de la clase política, el siguiente cuestionamiento es: ¿tiene usted algún proyecto de vida? El 88,1\% de los estudiantes encuestados del área metropolitana de Cúcuta y de Bucaramanga respondieron sí (Ver Tabla 6). Y frente a la pregunta: ¿qué tan satisfecho está usted con la educación recibida? El 63\% de los estudiantes del área metropolitana de Bucaramanga respondieron satisfechos y muy satisfechos. El 24,6\% afirmaron estar regularmente satisfechos. El 53,9\% de los estudiantes encuestados del área metropolitana de Cúcuta respondieron estar satisfechos y muy satisfechos, el 22,5\% regularmente satisfechos. Es decir, la percepción sobre la educación en ambas áreas metropolitanas es buena.

El siguiente interrogante fue: ¿cuál es su proyecto de vida? El $25,7 \%$ y el $20,2 \%$ de los estudiantes de último semestre universitario del área metropolitana de Bucaramanga y de Cúcuta, respectivamente, no respondieron. Pero, a pesar de ese alto porcentaje de abstinencia la tendencia fue ser profesionales y emprendedores. La razón principal de ser emprendedores es motivada por el alto ingreso de venezolanos al territorio que estimulan la demanda y la producción empresarial de productos.

Paradójicamente, la situación política de Venezuela estimula el mercado, de allí que los Santanderes ofrezcan el más alto resultado de investigación en jóvenes emprendedores, incluso muy por encima de lo que respondieron los estudiantes de último semestre del Valle de Aburrá.

El porcentaje de estudiantes de último semestre que quieren ser profesionales es casi igual al de emprendedores en ambas áreas metropolitanas (Ver Tabla 6). Lo más sorprendente, en términos de equidad de género, es que en igual porcentaje, tanto los hombres como las mujeres quieren ser emprendedores. Ante la pregunta, ¿creen ustedes que pueden realizar su proyecto de vida en sus municipalidades? En igual porcentaje, tanto el 60,2\% de los hombres como el 60,1\% de las mujeres encuestadas respondieron que sí.

La pregunta siguiente fue: ¿dejaría su municipio 
para realizar su proyecto de vida? El 85,4\% de los hombres y el 79,4\% de las mujeres encuestadas afirmaron que Sí. Las razones principales para dejar la municipalidad fueron: Bajo desarrollo municipal en un 15\% e insatisfacción por el territorio en un 11,6\% (Ver Tabla 6). Los estudiantes que manifiestan quedarse, la razón principal fue el desarrollo municipal en un $16,1 \%$ y el amor al territorio en un $8,2 \%$.
Por otro lado, los estudiantes de último semestre que afirman dejar sus municipalidades para hacer realidad su proyecto de vida el $49 \%$ de los encuestados de Bucaramanga y el $65 \%$ de los futuros profesionales de Cúcuta afirman regresar a trabajar y a vivir. Es decir, el área metropolitana de Cúcuta es la ciudad colombiana que más retiene a la población profesional que forma.

Tabla 6. Santanderes. Realización del proyecto de vida

\begin{tabular}{|c|c|c|c|c|c|}
\hline $\begin{array}{c}\text { ¿Posee usted algún proyecto } \\
\text { de vida? }\end{array}$ & \multicolumn{2}{|c|}{ Hombres } & \multicolumn{2}{|r|}{ Mujeres } & Total \\
\hline NO & \multicolumn{2}{|c|}{$15.5 \%$} & \multicolumn{2}{|r|}{$7.8 \%$} & $11.9 \%$ \\
\hline $\mathrm{SI}$ & \multicolumn{2}{|c|}{$84.5 \%$} & \multicolumn{2}{|r|}{$92.2 \%$} & $88.1 \%$ \\
\hline Tipo de proyecto de vida & Profesional & Familiar & Laboral & Emprendimiento & $N / R$ \\
\hline Bucaramanga & $36.5 \%$ & $5.0 \%$ & $1.0 \%$ & $31.7 \%$ & $25.7 \%$ \\
\hline Cúcuta & $31.9 \%$ & $12.8 \%$ & $2.1 \%$ & $33.0 \%$ & $20.2 \%$ \\
\hline ¿Por qué Sí? & \multicolumn{2}{|c|}{ Hombres } & \multicolumn{3}{|c|}{ Mujeres } \\
\hline Adecuado desarrollo municipal & \multicolumn{2}{|c|}{$15.9 \%$} & \multicolumn{3}{|c|}{$18.4 \%$} \\
\hline Amor por el territorio & \multicolumn{2}{|c|}{$10.3 \%$} & \multicolumn{3}{|c|}{$5.8 \%$} \\
\hline Buena infraestructura educativa & \multicolumn{2}{|c|}{$9.9 \%$} & \multicolumn{3}{|c|}{$10.1 \%$} \\
\hline \multicolumn{6}{|l|}{ ¿Por qué NO? } \\
\hline Bajo desarrollo municipal & \multicolumn{2}{|c|}{$14.6 \%$} & \multicolumn{3}{|c|}{$15.5 \%$} \\
\hline Insatisfacción por el territorio & \multicolumn{2}{|c|}{$11.6 \%$} & \multicolumn{3}{|c|}{$11.6 \%$} \\
\hline No contesta & \multicolumn{2}{|c|}{$37.8 \%$} & \multicolumn{3}{|c|}{$38.6 \%$} \\
\hline \multicolumn{6}{|l|}{ ¿Volvería? } \\
\hline Trabajar & \multicolumn{2}{|c|}{$31 \%$} & \multicolumn{3}{|c|}{$47 \%$} \\
\hline Vivir & \multicolumn{2}{|c|}{$18 \%$} & \multicolumn{3}{|c|}{$18 \%$} \\
\hline Pasear & \multicolumn{2}{|c|}{$22 \%$} & \multicolumn{3}{|c|}{$17 \%$} \\
\hline No volvería & \multicolumn{2}{|c|}{$1 \%$} & \multicolumn{3}{|c|}{$2 \%$} \\
\hline No responde & \multicolumn{2}{|c|}{$28 \%$} & \multicolumn{3}{|c|}{$16 \%$} \\
\hline Total general & \multicolumn{2}{|c|}{$100 \%$} & \multicolumn{3}{|c|}{$100 \%$} \\
\hline
\end{tabular}

Fuente: Centro de estudios económicos regionales CEER de la ESAP y del grupo de investigación Gobierno, Territorio y Cultura de Antioquia. 2018.

Los estudiantes de último semestre del área metropolitana de Bucaramanga son los menos satisfechos con el territorio, el gobierno local y el progreso económico alcanzado, por lo tanto, el riesgo de pérdida de inversión social municipal es más alto, en otras palabras, la fuga de cerebros altamente calificado es mayor (aproximadamente un 51\%). Por el contrario, los estudiantes de último semestre 
universitario encuestados del área metropolitana de Cúcuta son los más satisfechos con su gobierno, están más optimistas con el desarrollo económico alcanzado en sus territorios, en consecuencia, el riesgo de pérdida de inversión social municipal es menor, lo que significa que la fuga de cerebros altamente calificado es mucho menor (aproximadamente de 35\%).

\section{Valle de Aburrá}

En las 10 ciudades del área metropolitana del Valle de Aburrá se realizaron 730 encuestas a jóvenes de último semestre universitario de universidades públicas y privadas. La ciudad de Medellín desde el 2016 viene calculando el Índice Integrado de Felicidad IFI, el cual se evalúa a través de ocho dimensiones y 22 categorías la felicidad subjetiva de los medellinenses, a saber: Vida: en ella se miden estándares de calidad de vida, satisfacción con la vida, experiencias emocionales, usos del tiempo libre e igualdad de oportunidades. Experiencia de Felicidad: se mide las experiencias de felicidad sentidas por la ciudadanía y la distribución de la felicidad en términos de la equidad del gasto público. Entorno: participación cultural y deportiva, satisfacción con la oferta pública inmobiliaria o de equipamiento de ciudad, recursos naturales, espacio público. Salud: estado de salud y actividades saludables de la población, bienestar psicológico y valoración del sistema de salud por la ciudadanía. Seguridad y Convivencia: percepción ciudadana de la seguridad, victimización y convivencia. Educación: asistencia escolar y pertinencia de la educación. Buen gobierno y Ciudadanía activa: participación ciudadana, gobernanza, libertad de expresión, libertad de opinión y confianza en el gobierno. Movilidad Urbana: valoración de la movilidad peatonal y de vehículos [16-17]

Complementando el IFI, la Escuela Superior de Administración Pública construye el índice de retención de jóvenes talento en el área metropolitana del Valle de Aburrá a partir del estudio del proyecto de vida y el emprendimiento desarrollado en la investigación sobre la Felicidad Urbana [12], como aparece a continuación.
Satisfacción con la felicidad subjetiva

Para conocer qué tan feliz es el joven universitario de último semestre desde la construcción subjetiva de la felicidad, se indaga en ¿qué tan feliz se siente de vivir en el área metropolitana del Valle de Aburrá?

El $84,1 \%$ de los estudiantes universitarios hombres y el $80,3 \%$ de las estudiantes mujeres respondieron que están satisfechos y muy satisfechos. Es decir, aman el área metropolitana del Valle de Aburrá, su gran ciudad y municipios. El $11,2 \%$ de los hombres y el $13,9 \%$ de las mujeres son regularmente felices. Lo que implica que el $94,7 \%$ del total de los jóvenes universitarios del área metropolitana del Valle de Aburrá son felices de vivir en su ciudad. Solo el 5,2\% manifiesta estar insatisfechos y muy insatisfechos (Ver Tabla 7). Es relevante anotar que las mujeres son las que más insatisfacción muestran con la ciudad, si bien no se consultó cuáles son las razones, si es posible deducir, que se debe a la escasez de oportunidad laboral y el querer seguir viviendo dentro de la gran ciudad (Ver Tabla 9).

Tabla 7. Valle de Aburrá. Satisfacción de vivir allí.

\begin{tabular}{cccc}
\hline $\begin{array}{c}\text { ¿Qué tan } \\
\text { satisfecho está } \\
\text { usted de vivir } \\
\text { en el área } \\
\text { metropolitana? }\end{array}$ & Hombres & Mujeres & $\begin{array}{c}\text { Total } \\
\text { general }\end{array}$ \\
\hline 1 (Muy insatisfecho) & $3.1 \%$ & $3.7 \%$ & $3.4 \%$ \\
2 & $1.6 \%$ & $2.2 \%$ & $1.8 \%$ \\
3 & $11.2 \%$ & $13.9 \%$ & $12.4 \%$ \\
4 & $37.1 \%$ & $31.2 \%$ & $34.4 \%$ \\
5 (Muy satisfecho) & $47.0 \%$ & $49.1 \%$ & $47.9 \%$ \\
\hline Total general & $\mathbf{1 0 0 . 0 \%}$ & $\mathbf{1 0 0 . 0} \%$ & $\mathbf{1 0 0 . 0 \%}$ \\
\hline
\end{tabular}

Fuente: Centro de estudios económicos regionales CEER de la ESAP y del grupo de investigación Gobierno, Territorio y Cultura de Antioquia. 2018

El $85,9 \%$ de los jóvenes estudiantes de último semestre hombres y el $87,7 \%$ de las mujeres son felices y muy felices. Las razones principales de sus felicidades son: la 
satisfacción personal, es decir, porque ellos ven cumplir sus sueños, sus anhelos de autorrealización -los hombres en un 16,18\% y las mujeres en un 11,72\%-. Por bienestar físico y emocional, que implica la sociabilidad con los otros y con el entorno -los hombres en un 7,53\% y las mujeres en un 5,03\%-. Es notable, que para los jóvenes no prima las riquezas materiales sino los logros de superación y de bienestar emocional que le brinda el entorno (Ver Tabla 8).

Tabla 8. Valle de Aburrá. Felicidad subjetiva

\begin{tabular}{cccccc}
\hline \multicolumn{2}{c}{ ¿Qué tan feliz es usted? } & \multicolumn{3}{c}{ ¿Por qué es feliz? } \\
\hline & Hombres & Mujeres & & Hombres & Mujeres \\
\hline $\mathbf{1}$ (Poco feliz) & $0.5 \%$ & $2.8 \%$ & Bienestar físico y emocional & $7.53 \%$ & $5.03 \%$ \\
$\mathbf{2}$ & $2.6 \%$ & $0.6 \%$ & Bienestar material & $1.12 \%$ & $2.09 \%$ \\
$\mathbf{3}$ & $11.0 \%$ & $8.7 \%$ & No contesta & $24.97 \%$ & $22.45 \%$ \\
$\mathbf{4}$ & $34.2 \%$ & $38.3 \%$ & Relaciones familiares & $4.04 \%$ & $4.88 \%$ \\
$\mathbf{5}$ (Muy feliz) & $51.7 \%$ & $49.5 \%$ & Satisfacción personal & $16.18 \%$ & $11.72 \%$ \\
\hline Total general & $100.0 \%$ & $100.0 \%$ & Total general & $53.84 \%$ & $46.16 \%$ \\
\hline
\end{tabular}

Fuente: Centro de estudios económicos regionales CEER de la ESAP y del grupo de investigación Gobierno, Territorio y Cultura de Antioquia. 2018.

Sin embargo, no deja de ser muy significativo que más del $22 \%$ de los jóvenes hombres y mujeres no respondieron. De igual manera, más del $49 \%$ de los jóvenes universitarios no respondieron la pregunta por qué admiran a esa persona o personaje, es decir, por qué cobra interés para ellos y su motivación para permanecer en el lugar (Ver Tabla 8).

\section{Satisfacción con el proyecto de vida}

El 70,9\% de los jóvenes universitarios del último semestre del área metropolitana del Valle de Aburrá poseen un proyecto de vida, el $24,1 \%$ no tienen un proyecto de vida y el $5 \%$ no respondió la pregunta, principalmente, mujeres. Sin embargo, son ellas quienes afirman tener un proyecto de vida; no obstante, irónicamente, manifiestan no tener uno.

Entre los proyectos de vida que reportan más frecuencia están: el 39,5\% ser profesionales; el 11,6\% ser emprendedores; el $8,2 \%$ poseen un proyecto laboral y un porcentaje muy representativo del $33,5 \%$ se abstuvo de responder la pregunta. El porcentaje de emprendedores, aunque es bajo es muy representativo, ya que puede llegar a ser parte del indicador anual de nuevas empresas registradas en Cámara de Comercio, así también, de la tasa de crecimiento anual de nuevas ideas de emprendimiento atendidas en los Parques de emprendimiento o de cultura emprendedora, INNova, Rutan; o simplemente de las tasas anuales de crecimiento industrial de la ciudad con fines de exportación. De hecho, puede llegar a ser un muy buen indicador de crecimiento industrial y laboral en la región.

Obsérvese que al discriminar por género la información recogida, persiste la tendencia en el hombre de ser empresario y emprendedor; la mujer, por el contrario, tiende a ser menos aventurera, más conservadora, es decir, asume menos riesgo, como lo muestra el estudio sobre la dignidad de género en el mercado laboral colombiano. De igual manera, se mantiene la tendencia observada "a mayor desarrollo local más hombres estudian; por el contrario, a menor desarrollo local más mujeres estudian". Tendencia que puede ser explicada por razones económicas y culturales: "el hombre como abastecedor económico del hogar" [18]. 
Tabla 9. Valle de Aburrá. Proyecto de vida.

\begin{tabular}{|c|c|c|c|c|c|c|}
\hline $\begin{array}{l}\text { ¿Posee usted algún proyecto } \\
\text { de vida? }\end{array}$ & \multicolumn{2}{|c|}{ Hombres } & \multicolumn{2}{|r|}{ Mujeres } & \multicolumn{2}{|c|}{$\begin{array}{l}\text { Total } \\
\text { general }\end{array}$} \\
\hline$N / R$ & \multicolumn{2}{|c|}{$3.6 \%$} & \multicolumn{2}{|r|}{$5.4 \%$} & \multicolumn{2}{|c|}{$5.0 \%$} \\
\hline $\mathrm{SI}$ & \multicolumn{2}{|c|}{$69.9 \%$} & \multicolumn{2}{|r|}{$72.8 \%$} & \multicolumn{2}{|c|}{$70.9 \%$} \\
\hline NO & \multicolumn{2}{|c|}{$26.5 \%$} & \multicolumn{2}{|r|}{$21.8 \%$} & \multicolumn{2}{|c|}{$24.1 \%$} \\
\hline Total general & \multicolumn{2}{|c|}{$100.0 \%$} & \multicolumn{2}{|r|}{$100.0 \%$} & \multicolumn{2}{|c|}{$100.0 \%$} \\
\hline Tipo de proyecto de vida & Profesional & Familiar & Laboral & Emprendimiento & $N / R$ & Total \\
\hline Hombres & $20.4 \%$ & $4.1 \%$ & $4.6 \%$ & $7.7 \%$ & $16.1 \%$ & $53.0 \%$ \\
\hline Mujeres & $19.4 \%$ & $3.1 \%$ & $3.8 \%$ & $4.3 \%$ & $16.4 \%$ & $47.0 \%$ \\
\hline ¿Volvería? & \multicolumn{2}{|c|}{ Hombres } & \multicolumn{2}{|c|}{ Mujeres } & \multicolumn{2}{|l|}{ Total } \\
\hline Trabajar & \multicolumn{2}{|c|}{$14.4 \%$} & \multicolumn{2}{|c|}{$10.5 \%$} & \multicolumn{2}{|l|}{$24.9 \%$} \\
\hline Vivir & \multicolumn{2}{|c|}{$14.0 \%$} & \multicolumn{2}{|c|}{$12.4 \%$} & \multicolumn{2}{|l|}{$26.4 \%$} \\
\hline Pasear & \multicolumn{2}{|c|}{$19.1 \%$} & \multicolumn{2}{|c|}{$15.6 \%$} & \multicolumn{2}{|l|}{$34.6 \%$} \\
\hline No volvería & \multicolumn{2}{|c|}{$1.5 \%$} & \multicolumn{2}{|c|}{$0.8 \%$} & \multicolumn{2}{|l|}{$2.3 \%$} \\
\hline No responde & \multicolumn{2}{|c|}{$4.3 \%$} & \multicolumn{2}{|c|}{$7.4 \%$} & \multicolumn{2}{|l|}{$11.7 \%$} \\
\hline Total general & \multicolumn{2}{|c|}{$53.3 \%$} & \multicolumn{2}{|c|}{$46.7 \%$} & \multicolumn{2}{|l|}{$100.0 \%$} \\
\hline
\end{tabular}

Fuente: Centro de estudios económicos regionales CEER de la ESAP y del grupo de investigación Gobierno, Territorio y Cultura de Antioquia. 2018.

Con base en el cuestionamiento: ¿qué tan satisfecho está usted con su proyecto de vida? El 96,2\% de los jóvenes universitarios de último semestre en el Valle de Aburrá manifiestan estar satisfechos y muy satisfechos. Del $80,5 \%$ de los jóvenes estudiantes de último semestre que afirman dejar el área metropolitana para realizar su proyecto de vida, el 59,5\% quieren regresar para vivir y para trabajar.

Esto significa que el área metropolitana genera con su accionar sentimientos de pertenencia (Ver Tabla 9). Incluso cuando se preguntó: ¿qué tan satisfecho se siente usted viviendo en el área metropolitana? el $47,9 \%$ de los jóvenes universitarios de último semestre afirmó sentirse muy satisfecho, el 34,4\% está satisfecho y el $12,4 \%$ regularmente satisfecho. Obsérvese que el $82,3 \%$ está plenamente satisfecho. Resultados de investigación que permiten inferir que los jóvenes universitarios creen en la gran ciudad llamada área metropolitana
Tabla 10. Valle de Aburrá. Satisfacción administrativa

\begin{tabular}{ccccc}
\hline $\begin{array}{c}\text { Satisfacción } \\
\text { con }\end{array}$ & $\begin{array}{c}\text { Gestión } \\
\text { del } \\
\text { Alcalde }\end{array}$ & $\begin{array}{c}\text { Clase } \\
\text { política }\end{array}$ & $\begin{array}{c}\text { Progreso } \\
\text { económi- } \\
\text { co }\end{array}$ & Policía \\
\hline 1 & $10.2 \%$ & $12.9 \%$ & $6.9 \%$ & $15,7 \%$ \\
2 & $8.3 \%$ & $10.5 \%$ & $6.7 \%$ & \\
3 & $30.8 \%$ & $33.4 \%$ & $33.5 \%$ & $36.1 \%$ \\
4 & $29.4 \%$ & $25.2 \%$ & $29.1 \%$ & $48,3 \%$ \\
5 & $21.3 \%$ & $18.0 \%$ & $23.9 \%$ & \\
\hline $\begin{array}{c}\text { Total } \\
\text { general }\end{array}$ & $100.0 \%$ & $100.0 \%$ & $100.0 \%$ & $100.0 \%$ \\
\hline
\end{tabular}

Fuente: Centro de estudios económicos regionales CEER de la ESAP y del grupo de investigación Gobierno, Territorio y Cultura de Antioquia. 1 poco satisfecho. 5 muy satisfecho. 3 regularmente satisfecho. 2018.

Tales sentimientos parecen ser fruto de las buenas 
buenas acciones políticas. Primero, ante la pregunta ¿cómo evalúa usted la calidad en los servicios de seguridad de la policía? El 48,3\% de los jóvenes universitarios de último semestre del área metropolitana afirman que es muy buena, es decir, prácticamente, la mitad de los universitarios confían en el papel de la policía; dato que es muy relevante porque facilita el progreso de la ciudad, la confianza social y ciudadana en la ciudad. Sin embargo, el 36,1\% de los estudiantes universitarios dicen que la calidad en las acciones de seguridad de la policía es regular. Obsérvese que la confianza en la policía es superior a la no confian$z a$, ya que el $13,3 \%$ dice que son malas y el $2,4 \%$ que no existen. En otras palabras, existe una confianza relativamente buena en las acciones de seguridad de la policía en el área metropolitana del Valle de Aburrá (Ver Tabla 10).

Segundo, el 53\% de los estudiantes de último semestre universitario encuestado afirman estar satisfechos y muy satisfechos con el progreso económico de la región. El 33,5\% califican de regular el progreso económico de los municipios del área. En otras palabras, el 86,5\% ven progreso económico (Ver Tabla 10).

Tercero, el 50,7\% califican la gestión de los alcaldes del área metropolitana de satisfactoria y muy satisfactoria, siendo las de menor satisfacción las de Barbosa y Bello; y las de mayor satisfacción Medellín, Sabaneta y Envigado; y las de regular satisfacción Itagüí, Caldas y Girardota. Para el 30,8\% de los jóvenes universitarios la gestión de sus alcaldes ha sido regular y para el $18,5 \%$ ha sido insatisfactoria y muy insatisfactoria, debido a los actos latentes de corrupción (Ver Tabla 10).

Cuarto, según lo observado en los estudios de otras áreas metropolitanas en Colombia y Provincias en Antioquia, se mantiene la siguiente correlación: dependiendo de la gestión de los alcaldes los jóvenes universitarios evalúan su satisfacción con la clase política. De allí que, los jóvenes de Barbosa y Bello afirmen estar insatisfechos con la clase política de los municipios; por el contrario, los de Medellín, Sabaneta y Envigado manifiesten estar satisfechos con los líderes políticos de sus municipios (Ver Tabla 10).
Incluso, el $71,5 \%$ de los jóvenes universitarios de último semestre afirman estar satisfechos y muy satisfechos con su vecindario. Dato de investigación que es muy significativo si se toma en cuenta los problemas de violencia que han vivido los diferentes barrios y comunas del área metropolitana del Valle de Aburrá. De hecho, el $64,8 \%$ de los estudiantes universitarios afirman estar satisfechos y muy satisfechos con la seguridad del barrio que habitan. El 26\% afirman estar regularmente satisfechos. Es decir, solo el 9,2\% manifiestan estar insatisfechos y muy insatisfechos. Esta información es relevante, toda vez que evidencia la confianza en la gran ciudad del área metropolitana (Ver Tabla 10).

\section{CONCLUSIONES}

El estudio concluye que si existe una relación positiva entre emprendimiento y felicidad como realización de los proyectos de vida que facilita el desarrollo local y la reducción de las migraciones de jóvenes talento en las áreas metropolitanas del Eje Cafetero, Santanderes y Valle de Aburrá; siendo la ciudades de Cúcuta, Medellín y Manizales las que más retienen el capital humano que forman. Hecho que se ve reflejado en los niveles de satisfacción de los jóvenes universitarios en relación con el desarrollo económico alcanzado en dichas localidades. Satisfacciones que a su vez poseen correlación estadística positiva con los buenos gobiernos locales y la clase política.

Es decir, a mayor satisfacción de los jóvenes universitarios con el desarrollo local, mayor es la satisfacción de los jóvenes con los gobiernos locales y, por tanto, mayor es la retención local de la población joven calificada MAC; hecho claramente evidenciado en las ciudades de Envigado, Sabaneta, Medellín, Pereira, Dos quebradas, Manizales y Cúcuta.

El estudio evidencia que las principales ciudades de las áreas metropolitanas, se conciben como una forma positiva de integración administrativa y económica intermunicipal capaz de retener la capacidad emprendedora de su población joven 
calificada. Son ejemplo administrativo a otras municipalidades de la región. Lo que invita a repensar el ordenamiento territorial como estrategia de integración interdepartamental para el fortalecimiento del desarrollo socioeconómico y el mejoramiento del bienestar general, con obras de infraestructura que faciliten la movilidad, el intercambio de conocimientos y la protección de los recursos naturales [19-20].

\section{REFERENCIAS}

[1] Bentham and Mill. (2003). The classical utilitarians. United Satates of America: John Troyer.

[2] J. Mill (2002). El Utilitarismo. Madrid, España: Alianza Editorial.

[3] S. Guisán (2004). La ética mira a la izquierda. Barcelona, España: Anthropos

[4] Acemoglu, Daron \& Robinson, James. A. (2012). Por qué fracasan los países. Los orígenes del poder, la prosperidad y la pobreza. Colombia. Deusto, segunda edición.

[5] P. Beytía, E. Calvo (2011). ¿Cómo medir la felicidad? Revista Claves de Política Pública, 4, 1-10. DOI: $\quad$ http://dx.doi.org/10.2139/ssrn.2302809

[6] Y. Noah (2015). De animales a dioses. Breve historia de la humanidad. Bogotá, Colombia: Debate.

[7] J. Jacobs (2011). Muerte y vida de las grandes ciudades. Madrid, España: Capitán Swin Libros.

[8] E. Dussel (1998). Ética de la liberación. En la edad de la globalización y de la exclusión. Madrid: Trotta

[9] G. Rey (2015) La felicidad si se puede medir. [Impreso].Portafolio, Especial. [Consultado 25 de julio]. Bogotá, Colombia.

[10] Aristóteles. (2018). Ética Nicómaco. Madrid, España: Alianza.

[11] J. Gehl (2010). Cities for People. Washington: Island Press.

[12] Á. Muñoz (2018). La felicidad urbana en el Chocó, Valle de Aburrá y sus Provincias, Golfo de Urabá, Magdalena Medio y Santanderes. Medellín: Dossier de investigación de la Escuela Superior de Administración Pública de Antioquia-Choco.
[13] V. Camps, S. Giner (2014). Manual de Civismo. Barcelona, España: Ariel.

[14] Cámara de Comercio de Cúcuta. (2018). Cúcuta cómo vamos. San José de Cúcuta: Encuesta de Percepción Ciudadana. www.cccucuta.org.co

[15] Observatorio en Comercio Internacional y Frontera. 2018. Boletín Económico y de Comercio Internacional. Norte de Santander, Colombia: Universidad Francisco de Paula Santander, Vol. 019.

[16] Plan de Desarrollo Municipio de Medellín (2016). Medellín cuenta con vos 2016-2019. Alcaldía de Medellín: Secretaria de Planeación Municipal. Índice de felicidad Integral para Medellín IFI.

[17] En Medellín así es la experiencia ciudadana de felicidad sentida (2017). Periódico de circulación diaria. Noticias de Medellín. [Consultado en El Mundo.Com. el 20 de marzo]. Antioquia, Colombia.

[18] Á. Muñoz (2014). "Gender Dignity in the Colombian Labor Market (2000-2013)". Open Journal in Political Science, Vol. 4 and No. 4, p.p. 195-207.

[19] Ley 1454. (2011). Normas orgánicas para la organización político administrativa del territorio colombiano. Bogotá: Diario Oficial No. 48.115. Congreso de la República de Colombia.

[20] COMPES 3918 (2018). Estrategia para la implementación de los Objetivos de Desarrollo Sostenible ODS en Colombia. Bogotá: Consejo Nacional de Política Económica y Social. Departamento Nacional de Planeación. 